\title{
Vulneranilidad de la población costera frente a la contaminación orgánica y microbiológica en Santa Elena-Ecuador, 2020
}

\author{
Vulneranility of the coastal population to organic and microbiological \\ contamination in the Playa la Carioca Santa Elena-Ecuador community, 2020
}

https://doi.org/10.52808/bmsa.7e5.612.024

Isabel Del Rocío Balón Ramos ${ }^{1 *}$ https://orcid.org/0000-0002-7237-4912

Dadsania Soraya Rodríguez Moreira ${ }^{1}$ https://orcid.org/0000-0001-5556-600X

Yadira Mónica Solano Verá ${ }^{1}$ https://orcid.org/0000-0003-2657-868X

Karla Soledad Ortiz Chimbo ${ }^{1}$ https://orcid.org/0000-0002-7432-8848

\section{RESUMEN}

La problemática de la contaminación marina y su marcada influencia en la salud de los ecosistemas costeros, está estrechamente relacionada con el aumento creciente de las poblaciones que habitan estas zonas y, de igual manera, con el incremento de las actividades domésticas, agrícolas e industriales que, por el mal manejo e inadecuado control de los desechos sólidos y líquidos, afectan el medio marino con significativas implicaciones a nivel ecológico, socioeconómico y de salubridad (Marín et al., 2005). Cada vez más la contaminación en el planeta abarca un tema importante de tratar, esto se debe al agotamiento de los recursos naturales y al calentamiento global que afecta a toda la humanidad de diferentes maneras. Vargas (2016) aclara que la sostenibilidad juega un papel fundamental que busca no poner en peligro al medio ambiente ni los recursos naturales que existen en el lugar. Con el propósito de evaluar los efectos e impactos que ocasionan la contaminación, la susceptibilidad al deterioro de funciones ecológicas, servicicos ecosistemicos, y la capacidad de adaptación, se desarrollo un análisis integrado de amenazas y vulnerabilidad ante la contaminación orgánica y microbiológica en la población costera de Santa Elena-Ecuador, 2020. La presente investigación permitio evidenciar el estado de vulnerabilidad de la localidad situada en zona costera de Santa Elena, ante la contaminación orgánica y microbiológica. Asimismo puede ser utilizada como soporte en la generación de planes y programas que ofrezcan para el control y disminución de la contaminación en los hogares como en la zona marino-costera, en la medida en que se identifican puntos clave para el fortalecimiento de la comunidad tanto en términos de infraestructura como el caso de los servicios de acueducto y alcantarillado así como de capital social y dependencia del uso de agua en la comunidad en estudio.

Palabras Clave: Contaminacion ambiental, Contaminación organica y microbiologíca en las costas de Santa Elena-Ecuador

\begin{abstract}
The problem of marine pollution and its marked influence on the health of coastal ecosystems, is closely related to the growing increase in the populations that inhabit these areas and, in the same way, with the increase in domestic, agricultural and industrial activities that, due to the mismanagement and inadequate control of solid and liquid waste, affect the marine environment with significant implications at an ecological, socioeconomic and health level (Marin et al., 2005). More and more pollution on the planet encompasses an important issue to deal with, this is due to the depletion of natural resources and global warming that affects all of humanity in different ways. Vargas (2016) clarifies that sustainability plays a fundamental role that seeks not to endanger the environment or the natural resources that exist in the place. In order to evaluate the effects and impacts caused by pollution, susceptibility to deterioration of ecological functions, ecosystem services, and adaptation capacity, an integrated analysis of threats and vulnerability to organic and microbiological contamination was developed in the coastal population of Santa Elena-Ecuador, 2020. The present investigation allowed showing the state of vulnerability of the locality located in the coastal zone of Santa Elena, in the face of organic and microbiological contamination. It can also be used as a support in the generation of plans and programs that offer for the control and reduction of pollution in homes and in the marine-coastal zone, to the extent that key points are identified for the strengthening of the community both in terms of infrastructure, such as the case of the aqueduct and sewerage services, as well as social capital and dependence on the use of water in the community under study.
\end{abstract}

Key Words: Environmental contamination, organic and microbiological contamination in the coasts of Santa Elena-Ecuador

1 Universidad Estatal Península de Santa Elena, Ecuador *Autor de correspondencia: balonramosisabeldelrocio@gmail.com

\section{Introducción}

La contaminación marina y su marcada influencia en la salud de los ecosistemas costeros, está estrechamente relacionada con el aumento creciente de las poblaciones que habitan estas zonas y, de igual manera, con el incremento de las actividades domésticas, agrícolas e industriales que, por el mal manejo e inadecuado control de los desechos sólidos y líquidos, afectan el medio marino con significativas implicaciones a nivel ecológico, socioeconómico y de salubridad (Marín et al., 2005). Las aguas costeras con fines recreativos como las playas, por lo general, se encuentran 
en las proximidades de las áreas urbanas, donde los vertimientos sin tratar, con altos contenidos de microorganismos patógenos y otros agentes contaminantes, representan uno de los principales problemas sanitarios y ecológicos de las zonas costeras (Garay et al., 2002). Cabe destacar que el Ecuador es el cuarto productor de hidrocarburos en América Latina y también es conocido por ser el país que acoge la biodiversidad más densa del planeta en referencia a su superficie (Dangles, 2009). Los recursos naturales pueden claramente ser considerados como importantes generadores de ingresos para una población, pero el poco conocimiento de las personas acerca de la riqueza de los mismos, conlleva a un escaso nivel de valoración, deficiente manejo para su utilización, por lo que genera un impacto negativo al medio ambiente y al desarrollo del lugar. Ecuador, un país considerado megadiverso y con importantes áreas naturales, son escasos y limitados los estudios e investigaciones que se han llevado a cabo con la finalidad de conocer el grado de importancia de los recursos naturales para las personas que habitan en estas áreas y hacen uso de los mismos (Quizhpe et al., 2017). En La OMT (2013) hace énfasis en que el desarrollo del turismo puede traer beneficios para los países en desarrollo, y un tipo de turismo esencial en los que se puede trabajar es el turismo sostenible. En Ecuador el turismo ocupa un puesto importante, constituyéndose en la tercera fuente de ingresos para el país. Además, se ha convertido en pieza fundamental para el mejoramiento de la calidad de vida de la población.

De acuerdo a Ferrán et al. (2016), es por estas razones que el Estado ecuatoriano creó la marca país "Ecuador ama la vida", impulsando una campaña de promoción turística no solo local sino internacional, con el objetivo de dar a conocer al resto del mundo sus riquezas naturales, socioculturales, religiosas y de biodiversidad, creando así una nueva perspectiva para nuevos proyectos turísticos, buscando inversionistas que reactiven este sector tan importante para la economía del país. Cada vez más la contaminación en el planeta abarca un tema importante de tratar, esto se debe al agotamiento de los recursos naturales y al calentamiento global que afecta a toda la humanidad de diferentes maneras. Vargas (2016) aclara que la sostenibilidad juega un papel fundamental que busca no poner en peligro al medio ambiente ni los recursos naturales que existen en el lugar. Por su parte, Hernanz \& Gil en el 2013, expresaron que el Estado ecuatoriano se encuentra sujeto a una nueva forma de desarrollo sociopolítico, cuyo enfoque principal ha sido la sostenibilidad, en este sentido se ha considerado al turismo sostenible y su desarrollo en un lugar preponderante dentro de la nueva Constitución de la República en donde existen leyes como la Ley de Aguas, Ley de Prevención y Control de la Contaminación Ambiental y sus reglamentos, Ley Forestal y de Conservación de Áreas Naturales y de Vida Silvestre, Ley de Hidrocarburos y su reglamento ambiental, Ley de Minería, que incluye aspectos relativos a la preservación del ambiente, Ley de Gestión Ambiental, la cual se refiere al desarrollo sostenible. Asimismo, se crearon derechos de la naturaleza y propuestas que se enfatizan en el Sumak Kawsay o "el Buen Vivir" cuyos objetivos son mantener una relación armoniosa entre los ejes social, político y ambiental que conforman la sostenibilidad, asegurando que las presentes y futuras generaciones puedan satisfacer sus necesidades. Con el propósito de evaluar los efectos e impactos que ocasionan la contaminación, la susceptibilidad al deterioro de funciones ecológicas, servicicos ecosistemicos, y la capacidad de adaptación, se desarrollo un análisis integrado de amenazas y vulnerabilidad ante la contaminación orgánica y microbiológica en la población costera de Santa Elena-Ecuador, 2020.

\section{Materiales y Métodos}

La estimación de la vulnerabilidad está ligada a los conceptos de amenazas (naturales, antrópicas y climatológicas) y elementos expuestos (la población humana y los ecosistemas) que son los insumos intrínsecos del análisis de riesgos, producto de la amenaza por la vulnerabilidad (Tejada et al., 2003).

\section{Area de estudio}

La investigación se ejecuto en las areas de Salinas, La Libertad, Ayangue, Punta Carnero y Punta Blanca, pertenecientes a la zona costera de Santa Elena-Ecuador. En este caso se analizó la vulnerabilidad usando, la amenaza por contaminación orgánica y microbiológica. La evaluación de la amenaza y vulnerabilidad por contaminación en zonas costeras se basó en el desarrollo de las etapas que se plantean en el esquema metodológico (Figura 2). Adicionalmente, se incluyó información sobre las fuentes de contaminación y el manejo de los residuos sólidos y vertimientos, principales fuentes de contaminación orgánica y microbiológica. Con la caracterización ambiental se obtuvo el diagnóstico, donde se identifican las fuentes de amenaza contaminante, los elementos expuestos a valorar, los indicadores y la escala de análisis. Con estos insumos se realizó el Análisis de Amenazas en función de la probabilidad (P) que es la posibilidad de que se presente una amenaza por contaminación sobre los elementos expuestos, la intensidad que es el grado de afectación de la amenaza sobre los elementos expuestos (I) y la extensión que es la distancia o área de influencia del impacto en relación con el entorno (E). Por cada uno de estos atributos se asignó una calificación en una escala de uno a cinco, siendo un (1) la calificación más baja y cinco (5) las más alta. Amenaza $=\mathrm{P}^{*} \mathrm{I} * \mathrm{E}$. Se establecio la importancia relativa de las fuentes de amenaza, su distribución y clasificación considerando los tipos de fuentes, la cercanía a los elementos expuestos. Además, se valoraron los impactos potenciales a través de la identificación, evaluación y tipificación de las causas y fuentes contaminantes. Para la Evaluación de la vulnerabilidad se utilizaron diversas variables e indicadores de presión y estado que corresponden a los factores que condicionan o 
determinan la contaminación y por ende su grado de amenaza sobre los elementos expuestos, teniendo en cuenta los enfoques ambiental y socioeconómico. Para la calificación de la vulnerabilidad con enfoque socioeconómico se utilizó el índice de capacidad adaptativa (CA) con tres dimensiones (socioeconómica, sociopolítica y socioecológica) para saber qué tan vulnerable es la población ante las amenazas que representa la contaminación orgánica y microbiológica. Primero se identificó la intensidad de estas amenazas, para describir qué tan capaz es la comunidad para adaptarse a los impactos de la contaminación, considerando que una comunidad con mayor capacidad de adaptarse, será menos vulnerable. Para definir la CA es necesario calcular los tres índices que se describen a continuación, los cuales se determinaron con los datos que se obtuvieron a través de encuestas que se aplicaron en las áreas de estudio de la zona costera de Santa Elena. El índice socioeconómico (SE) determina los recursos físicos y económicos con los cuales cuenta la población para adaptarse ante los efectos de la contaminación orgánica y microbiológica, e identifica la dependencia económica de la población frente a los recursos que puedan estar siendo afectados por este tipo de contaminación (Tabla 1).

\section{Tabla 1. Indicadores de la dimensión socioeconómica}

\section{Dimensión Socioeconómica}

Variables tenidas en cuenta para el análisis de la capacidad economica de las comunidades

Pobreza: Este índice de pobreza permite tener una aproximación al nivel de riqueza de los hogares y de la comunidad en conjunto, a través del análisis de las necesidades básicas satisfechas, la persección de calidad de vida que tienen los hogares sobre si mismos y el estilo de vida material el cual indica qué también viven las personas en relación a lascosas que poseen

Características ocupacionales: características del mercado laboral en la comunidad, esto permite ver la facilidad o dificultad para cambiar de actividad económica si esta es altamente afectada por la contaminación

Infraestructura comunitaria: bienes y servicios públicos con los que cuenta la comunidad Fuente: Vivas et al., 2021.

El índice sociopolítico (SP) permite conocer los vínculos de las personas con las instituciones, tanto formales como informales, y analizar cómo se encuentra constituido su capital social, de tal forma que se puedan evaluar las fortalezas o debilidades dentro de la comunidad a la hora de gestionar estrategias en favor de la disminución de la contaminación y el manejo de los residuos (Tabla 2). El índice socioecológico (SEC) permite conocer la relación entre las comunidades y su entorno natural, y la capacidad de adaptarse a los efectos negativos de la contaminación.Con los resultados de estos tres índices, se determinó el nivel de capacidad de adaptación de la comunidad en una escala de cinco categorías: muy bajo (0-20), bajo (21-40), moderado (41-60), alto (61-80) y muy alto (81-100), para reflejar qué tan vulnerable son los hogares ante la contaminación orgánica y microbiológica de la zona costera de Santa Elena. Los valores de cada dimensión analizada (socio-económica, socio-política y socio-ecológica) resultaron del promedio de sus indicadores simples, mientras que el índice de capacidad adaptativa agregado se obtuvo tomando como criterio el mínimo valor de los tres indicadores.

\section{Tabla 2. Indicadores de la dimensión sociopolítica}

\begin{tabular}{|c|c|c|c|c|c|}
\hline $\begin{array}{l}\text { Dimensión Socio-politica } \\
\text { Variables tenidas en cuenta para el análisis de la capacidad institucional de las comunidades }\end{array}$ & & & & & \\
\hline $\begin{array}{l}\text { Estructura del capital social: describe la estructura del capital social de la comunidad, es } \\
\text { decir qué tan organizada está la comunidad, a través dela análisis de variables como número } \\
\text { de organizaciones en la comunidad, participación de los hogares en iniciativas comunitarias } \\
\text { y conocimiento y expectativa de las redes de apoyo }\end{array}$ & Muy bajo & Bajo & Moderado & Alto & Muy alto \\
\hline $\begin{array}{l}\text { Conocimiento del capital social: grado de apropiación que tiene la comunidad sobre sus } \\
\text { organizaciones sociales y valores éticos; se basa en la descripción del nivel de apropiación } \\
\text { de valores como la solidaridad, la cooperación y la confianza }\end{array}$ & \multirow[t]{2}{*}{$0-20$} & \multirow[t]{2}{*}{$21-40$} & \multirow[t]{2}{*}{$41-60$} & \multirow[t]{2}{*}{$61-80$} & \multirow[t]{2}{*}{$81-100$} \\
\hline $\begin{array}{l}\text { Acceso a instituciones: deja ver la presencia de las instituciones en la comunidad y cómo } \\
\text { estas son percibidas }\end{array}$ & & & & & \\
\hline
\end{tabular}

Fuente: Vivas et al., 2021.

Basados en los resultados de la calificación de los enfoques socioeconómico y ambiental se calculó la vulnerabilidad, sumando los valores obtenidos de cada conjunto de variables consideradas para cada elemento expuesto (Tabla 1), a éste resultado se le restó la capacidad adaptativa definida para los ecosistemas (GESAMP, 1996; PMRC, 2008; INVEMAR-TNC-CI-UAESPNN, 2009; PMRC, 2009), mientras que para la vulnerabilidad de la población se empleó la condición socioeconómica de cada área de estudio. Se realizo el estudio de vilnerabilidad, aplicando la siguiente ecuación: $V=\sum$ variables e indicadores - CA. Los resultados de vulnerabilidad se estandarizaron con base en la escala establecida de 1 a 5 , y los diferentes grados de vulnerabilidad de los ecosistemas y de la la contaminación orgánica y microbiológica (Tabla 3 ).

Tabla 3. Calificación de las variables e indicadores para determinar la vulnerabilidad de la población por Contaminación orgánica y microbiológica en zona Costera de Santa Elena, 2020

\begin{tabular}{|lc|}
\hline ELEMENTO EXPUESTO & 339 \\
\hline
\end{tabular}




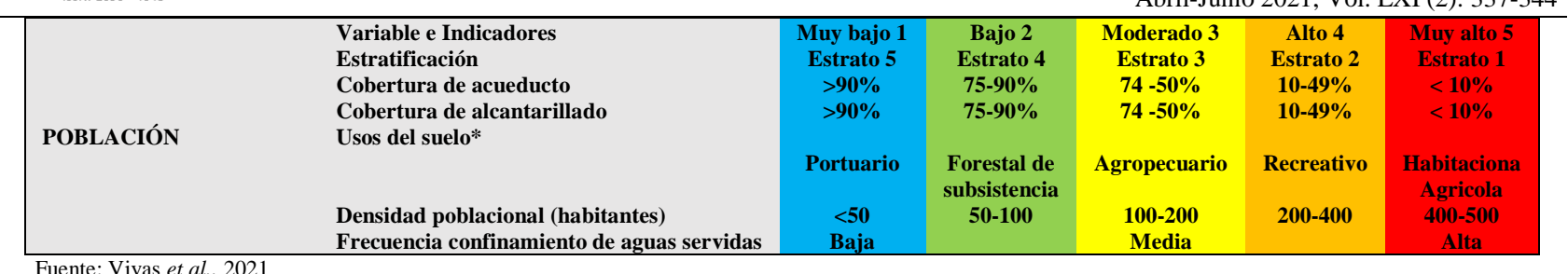

\section{Resultados}

Bajo esta perspectiva, el grado de amenaza por contaminación orgánica, se relaciono con el impacto de las actividades ejecutadas en las zonas estudiadas, se determino que en salinas, la amenaza de contaminación orgánica fue debida al aporte de residuos por dragado lo que represento un nivel 2,0, demostrando un bajo riesgo de amenaza por contaminación organica, en La Libertad y Punta Carnero, fue por aporte de sólidos organicos domésticos con nivel 3 de afectación con un riesgo alto, en las zonas de Ayangue y Punta Blanca, se determino un riesgo riesgo muy alto de amenaza, por aportes de residuos solidos organicos en zonas provenientes de la población, de las actividades tradicionales y de la actividad pesquera, con un nivel 5 de amenaza. Datos observados en la figura 1.

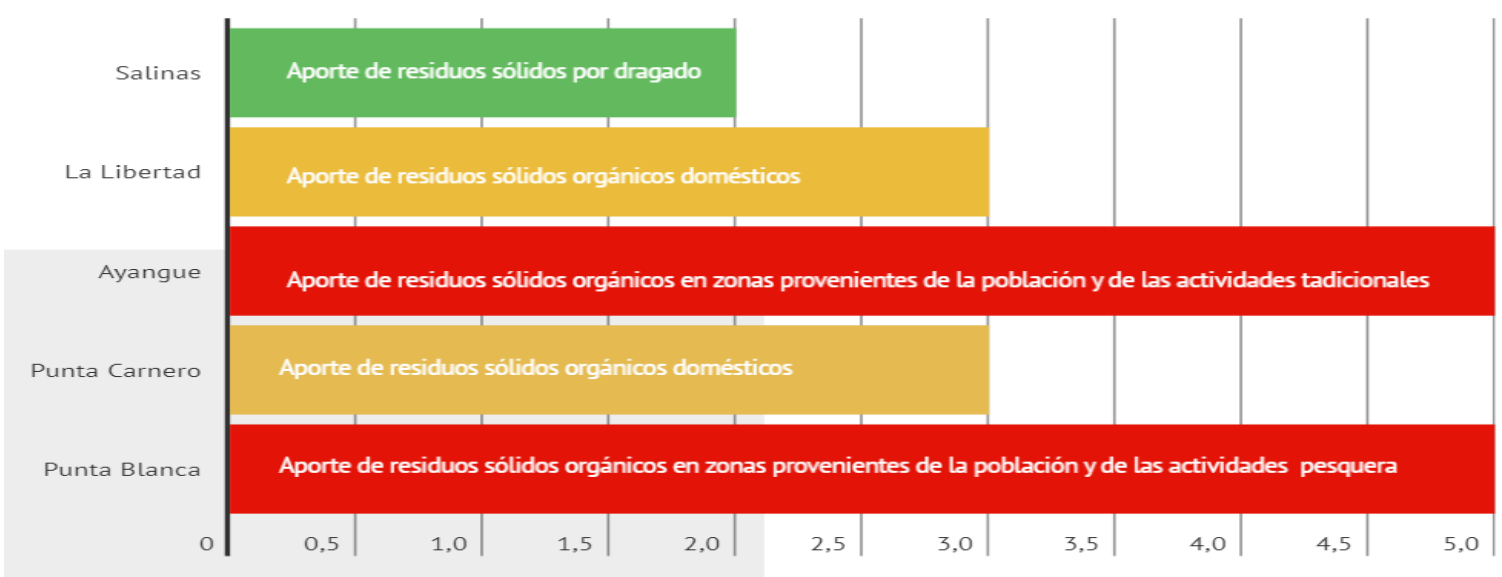

Figura 1. Amenazas por contaminación orgánica por comunidad en Santa Elena-Ecuador, 2020

En lo que respecta a la amenaza por contaminación microbiológica se puede evidenciar que, en el área de salina, este aporte fue por basura, con un nivel bajo de amenaza (2), en La Liibertad y Punta Carnero, fue debido a el aporte de vertimientos de aguas residuales por defectos de tubería y basura en la playa con un nivel alto (3), para la amenaza por contaminación microbiológica. Finalmete las áreas de Ayangue y Punta Blanca exhibieron una amenaza muy alta por contaminación microbiologíca (5), derivado de los vertimientos de aguas residuales domesticas asociado a baja cobertura de alcantarillado. Datos mostrados en la Figura 2.

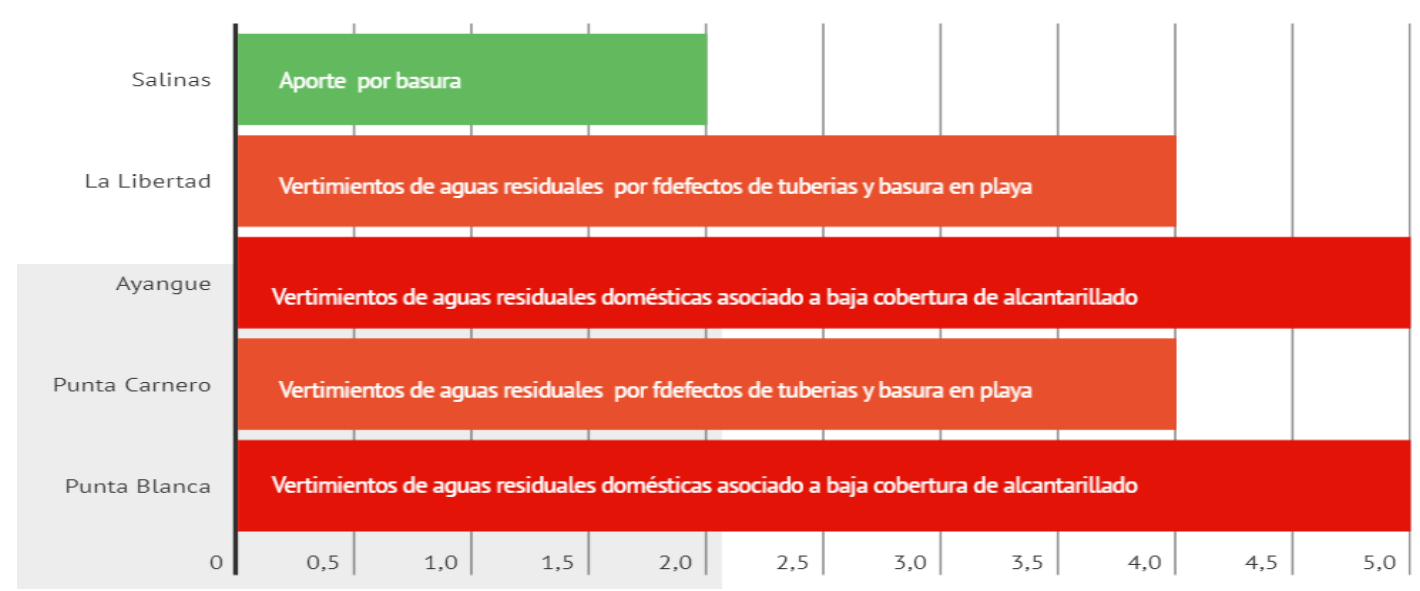

Figura 2. Amenazas por contaminación microbiológica por comunidades en Santa Elena-Ecuador, 2020 
En la zona Costera de Santa Elena en estudio, se realizó un análisis de capacidad adaptativa en el que se evidenció un nivel moderado de capacidad económica teniendo en cuenta que existe diversidad en las actividades económicas de las que dependen los hogares. A pesar de que existe un nivel de riqueza medio y un bajo nivel de infraestructura-comunitaria, por la falta de zonas de recreación y por la mala calidad de los servicios de alcantarillado, acueducto y recolección de residuos sólidos ( Tabla 4). En lo relativo a la capacidad adaptativa, se demostró la capacidad institucional, se observó una baja articulación con instituciones formales e informales especialmente de tipo ambiental. Las residencias tienen altas expectativas sobre estas redes, y esperan que solucionen gran parte de las problemáticas que se presentan. La seguridad, apoyo y colaboración son valores que permiten fortalecer el capital social en las comunidades; en relación a esto existe un bajo sentido de cooperación y confianza entre los miembros de la comunidad, mientras que la solidaridad entre vecinos es moderada. Es importante destacar, en relación con el agua contaminada, aprecian un nivel alto de contaminación, especialmente por la presencia de aguas residuales y desechos orgánicos en el agua.

Tabla 4. Capacidad adaptativa de las comunidades en Santa Elena-Ecuador, 2020

\begin{tabular}{|c|c|c|}
\hline \multicolumn{3}{|c|}{$\begin{array}{c}\text { Capacidad adaptativa } \\
\text { Baja capacidad para adaptarse por el deterioro de la infraestructura comunitaria y omisión de } \\
\text { la respuesta oficial } \\
(40)\end{array}$} \\
\hline & Dimensior & \\
\hline $\begin{array}{l}\text { para } \\
\text { de }\end{array}$ & $\begin{array}{r}\text { Poca acción o } \\
\text { capital social y } \\
\text { respec }\end{array}$ & $\begin{array}{l}\text { cimiento } \\
\text { atal y su }\end{array}$ \\
\hline
\end{tabular}

Según el estudio del grado de vulnerabilidad por contaminación orgánica, resulto ser muy alta (5) en el área de Ayangue y Punta Blanca $(4,5)$ ocasionada por la mayor concentración de la población insalubres, sumado a la inadecuada deposición de residuos. En lo que respecta a Punta Carnero y La Libertad, el grado de vulnerabilidad por contaminación orgánica, resulto ser alta con un nivel de 4 y 3 respectivamente, originada por la inadecuada disposición de residuos. Por otra parte, la zona de Salinas arrojó una baja vulnerabilidad por contaminación orgánica (2) como resultado de una recolección periódica de los residuos. Datos señalados en la figura 3.

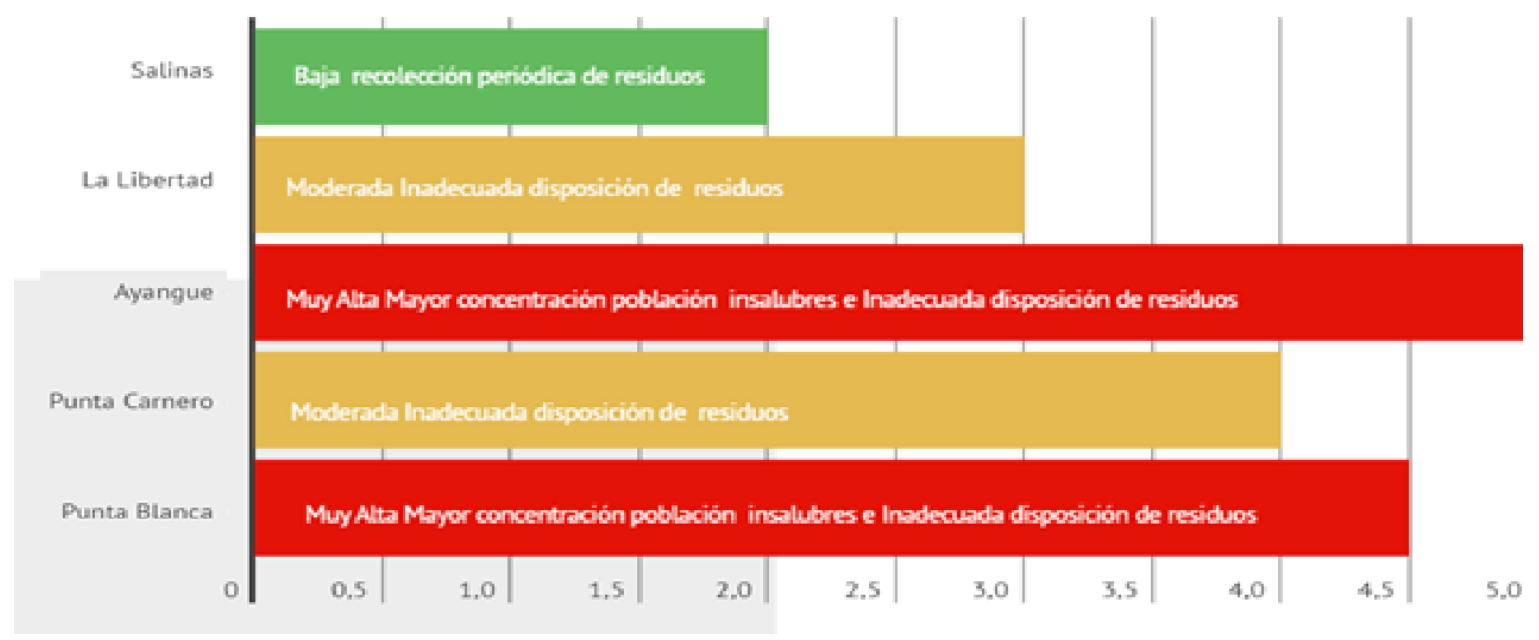

Figura 3. Grado de vulnerabilidad obtenido por contaminación orgánica por comunidad en Santa ElenaEcuador, 2020

Ayangue presento una muy alta vulnerabilidad por contaminación microbiológica (5) y Punta Blanca $(4,5)$, como consecuencia de una mayor concentración de la población y manejo inadecuado de los desechos y las aguas servidas. Asimismo, Punta Carnero y la Libertan presentaron una alta vulnerabilidad por contaminación microbiológica aunque en menor grado, 3,5 y 2,5 respectivamenta, ocasionada por una mayor concentración de la población y manejo inadecuado de los desechos y las aguas servidas. Finalmente en el área de salinas se evidencio una baja vulnerabilidad por contaminación microbiológica (2) por fallas en el servicio de alcantarillado. Datos indicados en la figura 4. 


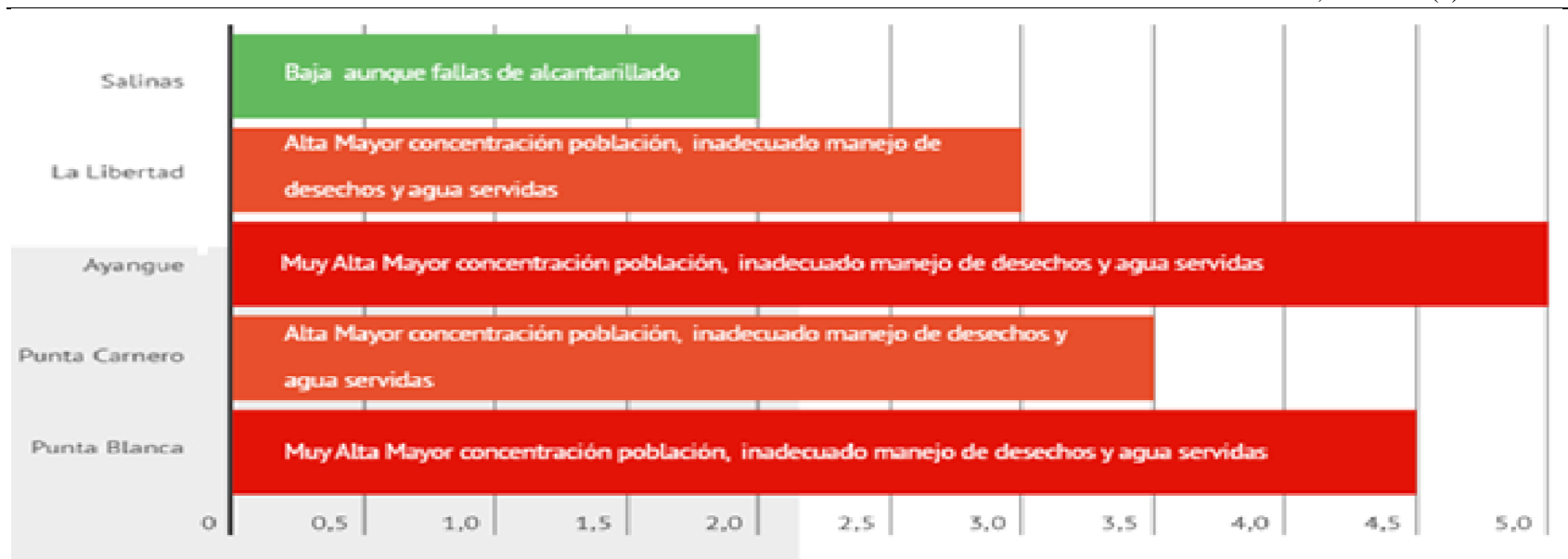

Figura 3. Grado de vulnerabilidad obtenido por contaminación microbiológica por comunidad en Santa ElenaEcuador, 2020

\section{Discusión}

Los hábitats costeros y marinos a nivel mundial han sido reconocidos como sistemas altamente productivos, con escasos niveles de protección y amenazados principalmente por las actividades humanas realizadas en tierra y en el mar (Garay et al., 2004; DNP, 2007). Las sustancias contaminantes que se integran al sistema por acciones antrópicas y por eventos naturales producen efectos nocivos sobre la salud humana y los ecosistemas de la zona, sedimentación entre otros efectos, convirtiéndolos en elementos vulnerables (Steer et al., 1997; Tejada et al., 2003), por esto se requiere adelantar estudios que permitan determinar la vulnerabilidad de los elementos expuesto frente a las amenazas latentes y plantear medidas eficaces que prevengan, reduzcan, mitiguen y controlen las fuentes que producen estos daños ambientales. El grado de amenaza por contaminación orgánica para la población estudida, estuvo relacionada con un riesgo alto por aporte de sólidos organicos domésticos, asimismo, con un riesgo muy alto, por aportes de residuos solidos organicos en zonas provenientes de la población, de las actividades tradicionales y de la actividad pesquera.

Los vertimientos de aguas residuales por defectos de tubería y basura en la playa con un nivel alto, y un riesgo muy alto, por aportes derivado de los vertimientos de aguas residuales domesticas asociado a baja cobertura de alcantarillado, en lo que respecta a la amenaza por contaminación microbiológica. De las evidencias anteriores, se derivan las Enfermedades Diarreicas Agudas - EDA y el cólera (Garay y Vélez, 2004). Esto aumenta con la disposición inadecuada delos residuos sólidos producidos a diario por las poblaciones ubicadas en zonas de bajamar, donde la empresa de aseo no puede cumplir eficientemente su labor, debido a las dificultades de acceso (SSPD, 2014). La capacidad de adaptarse ante perturbaciones generadas por las amenazas de contaminación orgánica y microbiológica es baja teniendo en cuenta que el mínimo valor de los tres índices o dimensiones descritas anteriormente, fue de 40 para el índice socio-político. Este análisis permite ver que es importante que las comunidades se fortalezcan como instituciones no formales lo que propiciaría un acercamiento más efectivo con instituciones formales especialmente las ambientales y de esta forma generar estrategias para mejorar su infraestructura comunitaria y con ello su capacidad de adaptarse.

El grado de vulnerabilidad por contaminación orgánica, resulto ser muy alta en el área de Ayangue y Punta Blanca ocasionada por la mayor concentración de la población insalubre, sumado a la inadecuada deposición de residuos. En lo que respecta a Punta Carnero y La Libertad, el grado de vulnerabilidad por contaminación orgánica, resulto ser alta, originada por la inadecuada disposición de residuos. Hallazgo que es similar al descrito por CIAM, 2008 e INVEMAR, 2009, además el grado de vulnerabilidad alto, por el aporte de residuos solidos y liquidos, que puede causar modificaciones importantes a la salud de la población estudiada y daños al ecosistema (Steer et al., 1997; Tejada et al., 2003; Steer et al., 2008; Fajardo, 2013). Los vertimientos industriales, son sustancias que se almacenan en los fondos donde gran parte de los organismos que lo forman son de importancia comercial y ejerce un efecto sobre los ciclos de vida de otras especies como los peces demersales que constituyen un recurso importante en el renglón de la economía en países costeros (Alongi, 1989). Estos aportes pueden influir además en la descomposición de los detritos, el reciclaje de nutrientes y el flujo de energía a niveles tróficos superiores (Cooksey et al., 2004).

En Ayangue y Punta Blanca, exhibieron una vulnerabilidad muy alta por contaminación microbiológica, como consecuencia de una mayor concentración de la población y manejo inadecuado de los desechos y las aguas servidas. Asimismo, Punta Carnero y la Libertan presentaron una alta vulnerabilidad por contaminación microbiológica aunque en menor grado ocasionada por una mayor concentración de la población y manejo inadecuado de los desechos y las 
aguas servidas. Otros estudios señalan, una alta vulneranbbiliad por contaminación microbiologíca en donde las descargas de aguas residuales a través del alcantarillado sanitario son deficientes e inadecuadas, con la presencia de emisarios de emergencia, que se hacen en forma directa sin ningún tratamiento por las comunidades palafíticas (Steer $e t$ al., 2008). Esta situación y el régimen de circulación de corrientes, favorecen la permanencia de coliformes que alteran la calidad del agua, este resultado ha sido corroborado por los diferente estudios realizados en zonas costeras (Collazos, 2014; Steer et al., 2008; Tejada et al., 2003).

\section{Conflictos de intereses}

Ninguno para declarar.

\section{Agredecimento}

A nuestra alma mater.

\section{Referencias}

Alongi D. (1989). Ecology of tropical soft-bottom benthos: a review with emphasis on emerging concepts. Rev. Biolo. Trop. 37 (1): 85-100.

CIAM. (2008). Políticas Oceánicas-Costeras Nacionales. Presentación de la Comisión Interministerial sobre Asuntos del Mar (CIAM) en el Taller Nacional Del Subsistema De Áreas Marinas. Bahía de Caráquez 6 Y 7 de agosto de 2008 .

Collazos, M. (2014). Definición de objetivos de calidad de vertimientos en la bahía de Buenaventura. Desarrollo de la fase I. Tesis Ing. Sanit., Uni. Aut. Occid. 135 P.

Cooksey C., Hyland W., Balthis M., Fulton G., Fulton M., Scott G. \& Bearden D. (2004). Soft-Bottom Benthic Assemblages and Levels of Contaminants in Sediments and Biota at Gray's Reef National Marine Sanctuary and Nearby Shelf Waters off the Coast of Georgia (2000 and 2001). NOAA Technical Memorandum NOS NCCOS 6. 55 pp.Disponible en: https://nmsgraysreef.blob.core.windows.net/graysreef$\mathrm{prod} / \mathrm{media} / \mathrm{archive/science/publications/pdfs/i-06.pdf}$ (Acceso enero 2021)

Dangles O. (2009). Biota maxima, Quito: PUCE-IRD.

DNP-Departamento Nacional de Planeación. (2007). 2019 Visión Colombia II Centenario. Aprovechar el territorio marino-costero en forma eficiente y sostenible. Propuesta para discusión. Excelsior impresores. DDTS-DNP. Bogotá.100P.

Ecuador. (2008). Decreto ${ }^{\circ}$ 1254. (2013). Decreto No 1.254 - Suprime el Programa de Manejo de Recursos Costeros (PMRC). Palacio Nacional en Quito. Disponible en: https://www.ecolex.org/es/details/legislation/decreto-no1254-suprime-el-programa-de-manejo-de-recursos-costeros-pmrc-lex-faoc084333/ (Acceso enero 2021)

Ecuador. (2009). Acuerdo Ministerial no 025 de fecha 10 demarzo del 2009, se expide el Reglamento Operativo del Proyecto de Manejo de Recursos Costeros-Etapa II (PMRC II).

Fajardo V. (2013). Capacitación técnica en el manejo de residuos sólidos y campañade sensibilización en la población de ladrilleros, pacífico valle caucano, Colombia. Tesis Admini. Amb., Uni. Aut. Occid. 190 P.

Ferrán Y., García L., Naranjo M.M. \& Marrero, M. (2016). La marca país (2010-2014): Ecuador ama la vida y su incidencia en la matriz productiva y el plan del buen vivir. UNIANDES EPISTEME Revista de Ciencia, Tecnología e Innovación 3(2):103-112.

Garay J., Marín B., Ramírez G., Betancourt J., Troncoso W. \& Gómez M. (2002). Diagnóstico y evaluación de la calidad ambiental marina en el Caribe y Pacífico colombiano. Red de vigilancia para la protección y conservación de la calidad de las aguas marinas y costeras. Diagnóstico INVEMAR.

Garay J. \& Vélez A. (2004). Programa Nacional de Investigación, Evaluación, Prevención, Reducción y Control de Fuentes Terrestres y Marinas de Contaminación al Mar-PNICM. Santa Marta: Instituto de Investigaciones Marinas y Costeras "José Benito Vives de Andréis"-INVEMAR. Santa Marta.134 P.

Garay-Tinoco, J.A., D.I. Gómez-López, \& Ortíz-Galvis J.R. (2006). Diagnóstico integral del impacto biofísico y socioeconómico relativo a las fuentes decontaminación terrestre en la bahía de Tumaco, Colombia y lineamientos básicos para un plan de manejo. Proyecto del Programa de las Naciones Unidas para el medio 
ambiente (PNUMA-Programa de Acción Mundial PAM) y comisión permanente del pacífico Sur CPPS. Instituto de Investigaciones Marinas y Costeras INVEMAR-Centro Control Contaminación del Pacífico CCCP-Corporación Autónoma Regional de Nariño CORPONARIÑO. SantaMarta, 290 P.

GESAMP. (1996). The contributions of science to coastal zone management. Grupo Mixto de expertos sobre los Aspectos Científicos de la Protección del Medio Marino (GESAMP). Roma, FAO. Reports and Studies, GESAMP 61:66 pp. URL: http://www.fao.org/docrep/meeting/003/w1639e/w1639e00.htm (Acceso enero 2021)

Hernanz I. \& Gil F. (2013). Reflexiones en torno del patrimonio para la sostenibilidad turística en áreas rurales. Boletin de interpretación numero 28.

INVEMAR-TNC-CI-UAESPNN. (2009). Informe Técnico: Planificación ecorregional para la conservación in situ de la biodiversidad marina y costera en el Caribe y Pacifico continental colombiano. En D. R.-Q.-T. Alonso. Santa Marta: INVEMAR. Serie de documentos generales No. 41.

Marín B., Garay J., Ramírez G., Betancourt J., Troncoso W. \& Gómez M. (2005). Diagnóstico y evaluación de la calidad ambiental marina en el Caribe y Pacífico colombiano red de vigilancia para la conservación y protección de las aguas marinas y costeras de Colombia. Diagnóstico Nacional y Regional. INVEMAR.

OMT. Organización Mundial de Turismo. (2013). Recuperado el 4 de diciembre de 2016. Disponible en OMT. Organización Mundial de Turismo. (2013). Recuperado el 4 de diciembre de 2016. Disponible en: https://www.unwto.org/es (Acceso enero 2021)

Quizhpe W., Veintimilla D., Aguirre Z., Jaramillo N., Pacheco E., Vanegas R. \& Jadán, O. (2017). Unidades de paisaje y comunidades vegetales en el área de Inkapirca, Saraguro, Loja, Ecuador. Bosques Latitud Cero 7(1):02- 122.

Steer R., Arias F., Ramos A., Sierra-Correa P., Alonso D. \& Ocampo P. (1997). Documento Base para la elaboración de la "Política Nacional de Ordenamiento Integrado de las Zonas Costeras Colombianas". Documento de consultoríapara el Ministerio del Medio Ambiente. Serie publicaciones especiales No.6. Santa Marta.375 P.

Superintendencia de Servicios Públicos Domiciliarios delegada para Acueducto, Alcantarillado y Aseo - SSPD. (2014). Informe ejecutivo de gestión Buenaventura Medio Ambiente S.A E.S.P Análisis 2013-2014. Bogotá. 12 P.

Tejada C., Castro L., Navarrete A., Cardona T. \& Otero L. (2003). Panorama de la Contaminación Marina del Pacífico Colombiano. San Andrés de Tumaco: Centro Control Contaminación del Pacífico Colombiano. Ed. DIMAR.Serie de Publicaciones Espaciales. 3: 120.

Vargas C. (2016). Importancia de la sostenibilidad ambiental en la ingeniería. Recuperado el 4 de diciembre de 2016. Disponible en: https://scielo.conicyt.cl/pdf/riat/v14n1/0718-235X-riat-14-01-00065.pdf (Acceso enero 2021) 